\title{
Concurrent weekly single cisplatin vs triweekly cisplatin alone with radiotherapy for treatment of locally advanced cervical cancer: a meta-analysis
}

This article was published in the following Dove Press journal: Cancer Management and Research

\author{
Jiahao Zhu ${ }^{1,2, *}$ \\ Shengjun $\mathrm{ji}^{1, *}$ \\ Qunchao $\mathrm{Hu}^{\prime}$ \\ Qingqing Chen' \\ Zhengcao Liu' \\ Jinchang $\mathrm{Wu}^{\prime}$ \\ $\mathrm{Ke} \mathrm{Gu}{ }^{\prime}$
}

'Department of Radiation Oncology, The Affiliated Suzhou Hospital of Nanjing Medical University, Suzhou, China; ${ }^{2}$ Department of Oncology, Nanjing Medical University, Nanjing, China

*These authors contributed equally to this work
Correspondence: Ke Gu

Department of Radiation Oncology, The Affiliated Suzhou Hospital of Nanjing Medical University, 16 West Baita Road, Suzhou 21500I, China

Tel +8651262364043

Fax +8651267294553

Email drguke@।63.com
Background: Radiotherapy (RT) concurrent with cisplatin (CDDP) is the standard regimen used for treatment of locally advanced cervical carcinoma. In this meta-analysis, we compared the weekly and triweekly single CDDP concomitant chemoradiation regimens for treatment of cervical cancer with respect to compliance, recurrence, survival, and acute adverse effects. Materials and methods: A systematic search for relevant studies was conducted in PubMed, Cochrane Library, EMBASE, and Medline databases. Fixed- or random-effects model was used for pooled analysis. The end points were overall survival, recurrence, compliance, and acute adverse effect reported as odds ratios (ORs) and 95\% CIs.

Results: Six randomized trials and two retrospective studies qualified the inclusion criteria. The regimen of triweekly CDDP alone concurrent with RT showed better compliance (OR, $0.49 ; 95 \%$ CI, $0.29-0.83 ; P=0.009)$. No significant difference was observed between the 2 arms with respect to recurrence, survival, and acute adverse effects (all $P>0.05$ ). However, triweekly CDDP regimen was associated with significantly lower incidence of local recurrence $(\mathrm{OR}, 1.83$; 95\% CI: 1.12-3.01; $P=0.02)$, while weekly CDDP regimen was associated with a lower risk of leucopenia (OR, 0.30; 95\% CI: 0.10-0.92; $P=0.03$ ).

Conclusion: Triweekly single platinum chemotherapy plus concurrent RT was superior to weekly CDDP regimen with respect to local recurrence and treatment compliance in patients with locally advanced cervical carcinoma.

Keywords: locally advanced cervical carcinoma, chemoradiotherapy, cisplatin, weekly, triweekly

\section{Introduction}

Cervical cancer (CC) is the fourth most commonly diagnosed cancer and the fourth leading cause of cancer deaths among women worldwide. An estimated $90 \%$ of deaths from $\mathrm{CC}$ occur in the developing countries. ${ }^{1}$ The application of human papillomavirus vaccine and advances in screening technology have contributed to the great achievements in prevention and treatment of $\mathrm{CC}$ and premalignant disease; however, the worldwide survival and prognosis of this malignancy is still very poor, especially for locally advanced cervical carcinoma (LACC). Based on favorable outcomes in 5 randomized clinical trials (RCTs), cisplatin (CDDP)-based chemoradiotherapy is strongly recommended for patients with LACC who require radiotherapy (RT). ${ }^{2-6}$ Furthermore, weekly CDDP regimen concurrent with radiation is widely accepted due to better compliance and low toxicity, compared with triweekly CDDP plus 5-fluorouracil (5-FU) regimen among these 5 studies. In a study by Lanciano et al, ${ }^{7}$ outcomes in the 5 -FU treatment arm were not superior to those in the weekly CDDP arm. Therefore, vari- 
ous chemotherapeutic combinations based on CDDP should be tried for concurrent chemoradiationtherapy (CCRT) in patients with CC. Moreover, comparing the alternative CDDP dose and dosing schedules in chemotherapy concurrent with RT is necessary.

In a meta-analysis by Petrelli et al, single CDDP and CDDP-based doublet chemotherapy (including 5-FU, hydroxyurea, cyclophosphamide, paclitaxel, and docetaxel) combined with RT were compared in the treatment of patients with CC. They found that platinum-based doublet chemotherapy plus concurrent RT increased the overall survival (OS) and progression-free survival (PFS) compared with RT plus single platin. Therefore, platinum-based combination therapy plus RT should be preferred over single platin plus RT for CC. In addition, regimen of CDDP plus 5-FU is recommended by leading guidelines among the various polychemotherapies. ${ }^{8}$ However, 2 of the previous 8 clinical trials adopted weekly CDDP, while 5 trials used triweekly CDDP, and the other 2 trials employed 4-weekly agent as the chemotherapeutic combinations. The optimal chemotherapy regimen based on CDDP is yet to be established.

$\mathrm{Hu}$ et al conducted a meta-analysis to evaluate the efficacy of weekly and triweekly CDDP with RT for treatment of CC. They found that weekly CDDP was associated with a lower risk of hematological toxicity compared with the triweekly CDDP with CCRT. However, the 2 regimens were comparable with respect to PFS and OS $(P>0.05),{ }^{9}$ which is similar to the results of another meta-analysis conducted by Chen et al. ${ }^{10}$ Nevertheless, most regimens of triweekly CDDP were combined with other chemotherapeutic drugs (such as tirapazamine, 5-FU, hydroyurea, paclitaxel) in both the meta-analyses. Use of these drugs may have confounded the analysis and affected the validity of the results. Therefore, the real difference between the 2 regimens may still be unknown to us.

Several recent studies have compared the 2 different single CDDP schedules with respect to survival and incidence of adverse events. Ryu et al found triweekly CDDP CCRT had better 5-year OS and higher relatively completion rate of scheduled chemotherapy cycles, but less severe neutropenia compared with the conventional weekly CDDP regimen. ${ }^{11}$ Conversely, outcomes of weekly CDDP regimen for CCRT were found to be better with respect to hematological toxicity in a study conducted by Kinjyo et al in $2017 .{ }^{12}$ No definitive conclusions were drawn from these studies. Therefore, 6 randomized trials and 2 retrospective studies were included in our meta-analysis to explore the difference between the different CDDP-alone regimens for patients with LACC.

\section{Materials and methods}

\section{Search strategy}

The databases of PubMed, Cochrane Library, EMBASE databases, and Medline were searched by using the following key words, (Cisplatin or Platinum or cis-Platinum or Platinol or Platidiam or CDDP), (Uterine Cervical Neoplasms or Cervical Neoplasms or Cervix Neoplasms or Uterine Cervix Cancers or Cervix Cancers or Cervical Cancers), (Triweekly or Every 3 weeks or 3 weeks), (Per week or Every week or Weekly or Once a week), and (chemoradiotherapy or chemoradiation or radiochemotherapy or chemotherapy or RT, or radiation or electromagnetic radiation). Only those studies published from 1990 to December 29, 2017 in English were considered. References of the included studies and related citations was also checked manually for potentially relevant studies. Two independent investigators evaluated each study. A consensus should be reached by discussion or the third investigator to resolve the disagreements created between the 2 reviewers.

\section{Inclusion and exclusion criteria}

Studies were included in the analysis if: 1) they were randomized controlled trials or retrospective studies that compared triweekly single CDDP plus RT plus vs weekly single CDDP plus RT; 2) no evidence of para-aortic lymph node or distant metastasis on pretreatment imaging (stages I-IVA); and 3) the long-term OS and recurrence rate, including local and distance, were assessed as outcomes to measure the effect of the treatment. If studies were duplicates, the study with the most up-to-date results was included. Studies were excluded if patients had previous histories of chemotherapy or RT, or other factors seriously affecting the survival and treatment processes.

We used the revised Jadad scale to evaluate the quality of the randomized controlled trials included in the primary outcome analysis. An article of high quality scored 4-7 points. The Nottingham Ottawa Scale (NOS) was used to assess observational studies. On the basis of NOS criteria, studies are scored between 0 and 9 stars. Six stars or greater was considered to be sufficiently higher-quality studies. ${ }^{13}$

\section{Statistical analysis}

OS and recurrence rate, including the locoregional relapse rate and rate of distant metastasis were the primary end points, and appliance, acute adverse were secondary end points. RevMan 5.1 software (Cochrane Collaboration's Information 
Management System) was used to conduct this meta-analysis. Variables among studies with minimal heterogeneity were assessed by fixed-effect model/Mantel-Haenszel method, otherwise, random-effects model/DerSimonian-Laird method was used when calculating the odds ratios (ORs) and $95 \%$ CIs in the specific event. Funnel plots and Harbord tests were used to examine potential publication bias in the meta-analysis.

\section{Results}

\section{Study selection and characteristics}

The search initially yielded a total of 1896 citations. A total of 8 trials ${ }^{14-19}$ were included in this review after exclusion of studies that did not qualify the inclusion criteria, duplicate publications, review articles, and meta-analyses. Two trials were not included due to lack of availability of relevant data. The study selection criteria for this meta-analysis are illustrated in Figure 1.

Among the 8 publications considered in this analysis, there were 6 prospective randomized trials and 2 retrospective case series. The 8 studies with a combined sample size of 934 patients were conducted in the USA, Japan, India, Korea, and Romania and were published between 2007 and 2017. All the patients recruited in these studies were newly diagnosed as LACC and received primary radical CCRT. Out of the 934 patients, 436 patients received weekly CDDP-based chemotherapy concurrent with RT, while 498 patients received triweekly regimen. For the retrospective studies, the NOS grades were 6-7 stars (out of a maximum possible score of 9 stars). For all the 6 randomized studies, the overall quality described by Jadad scores was 3 out of 5. Table 1 shows the detailed analysis of the studies.

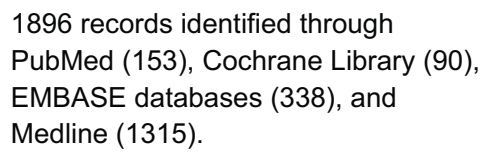

1860 studies were

excluded (duplicates, systematic review

evaluating meta-analysis, unrelated

topic, or not meet the treatment of concurrent chemoradiotherapy).

36 studies were accessed for eligibility.

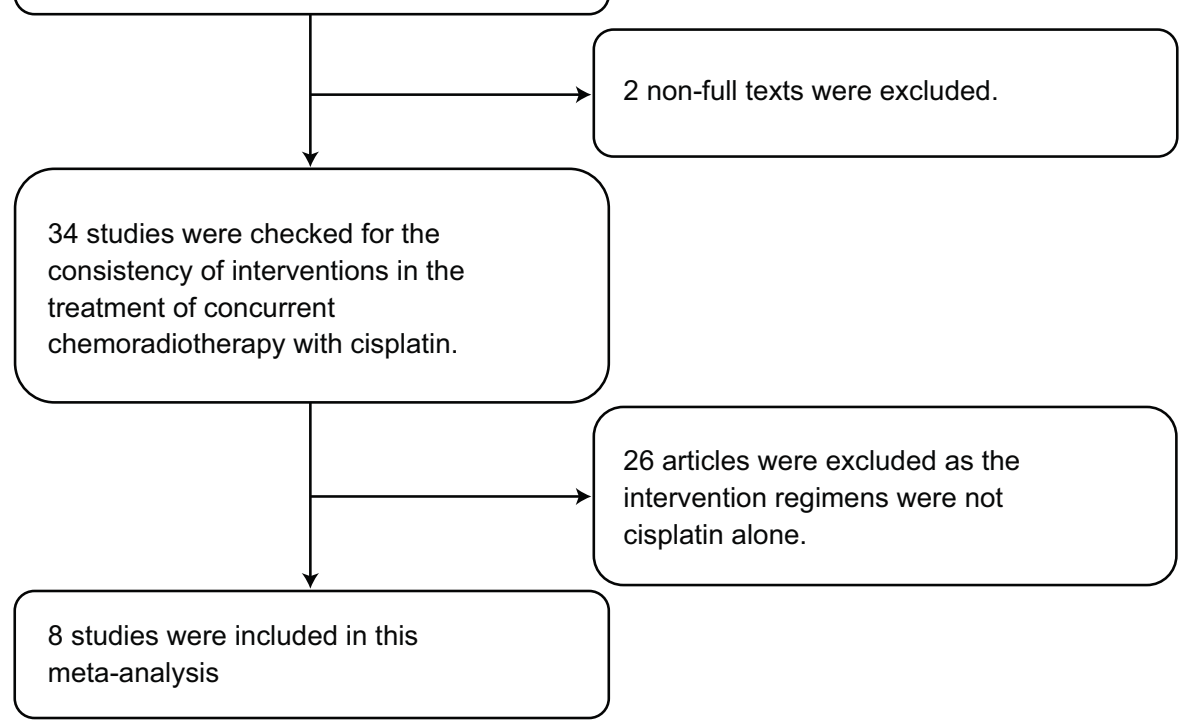

Figure I Study selection flow about the cisplatin-based chemoradiotherapy of cervix cancer. 


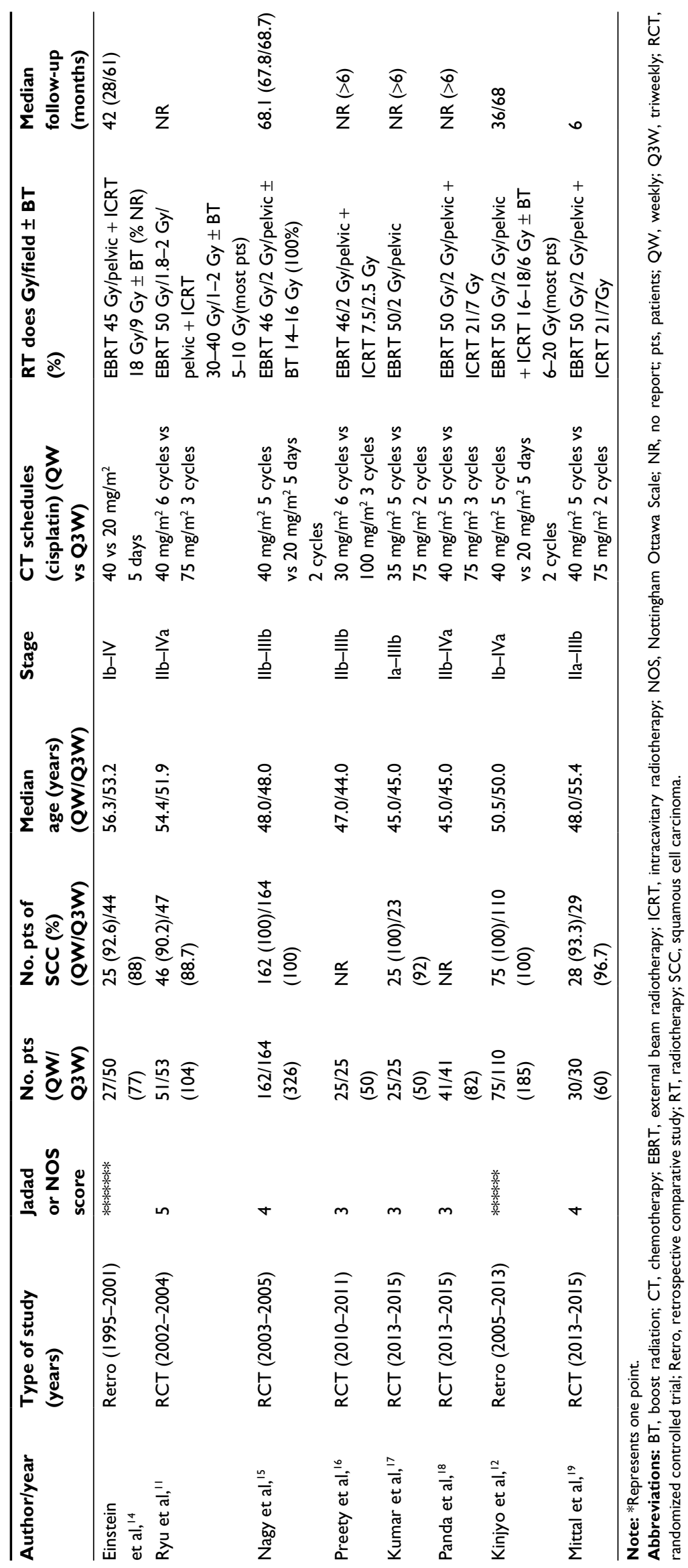




\section{Primary end points: 5-year OS and recurrence rate}

The meta-analysis of 5-year OS ( $\mathrm{n}=3$ studies) was affected by a relatively high heterogeneity among the trials $\left(I^{2}=58 \%\right)$. Therefore, the random-effects model was chosen for pooled analysis. The Harbord test showed lack of significant heterogeneity among the trials $(P>0.05)$. The analysis revealed no statistically significant difference between the triweekly and weekly regimens of CDDP-based chemotherapy plus RT with respect to 5-year OS (OR, 0.63; 95\% CI: 0.32-1.23; $P=0.17$; Figure 2).

For the meta-analysis of recurrence rate ( $\mathrm{n}=4$ studies), no significant heterogeneity was observed among the trials. Therefore, the fixed-effects model was chosen for pooled analysis. The study by Einstein et al showed no significant difference between the 2 regimens of CCRT with respect to 3 -year recurrence $(P=0.11) .{ }^{14}$ Analysis of data from the other 3 studies also showed no significant difference with respect to 5-year recurrence (OR, 1.41; 95\% CI: 0.99-2.01; $P=0.06$; Figure 3 ). We performed subgroup analysis in terms of 5-year recurrence and found that triweekly CDDP plus RT was associated with a $43 \%$ reduced risk of 5 -year local recurrence compared with that with weekly CDDP-based CT plus RT (OR, 1.83; 95\% CI: 1.12-3.01; $P=0.02$; Figure 4). However, no significant difference was observed between the 2 regimens of CCRT with respect to 5 -year distant recurrence (OR, 1.15; 95\% CI: 0.72-1.84; $P=0.57$; Figure 4).

\section{Secondary end points: compliance and acute adverse events}

Not completing the total cycles of chemotherapy or missing any dose of CDDP or delaying radiation period longer than a certain period of time, varieties existed in different studies, was defined as patients with bad compliance. The

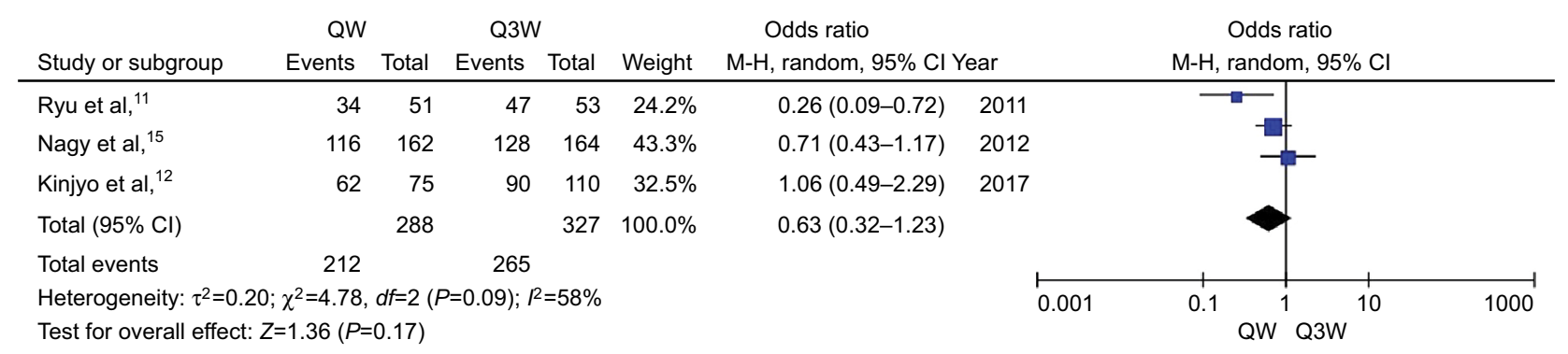

Figure 2 Meta-analysis evaluating 5-year OS of weekly single cisplatin or triweekly cisplatin alone combined with radiotherapy. Abbreviations: OS, overall survival; QW, weekly; Q3W, triweekly.

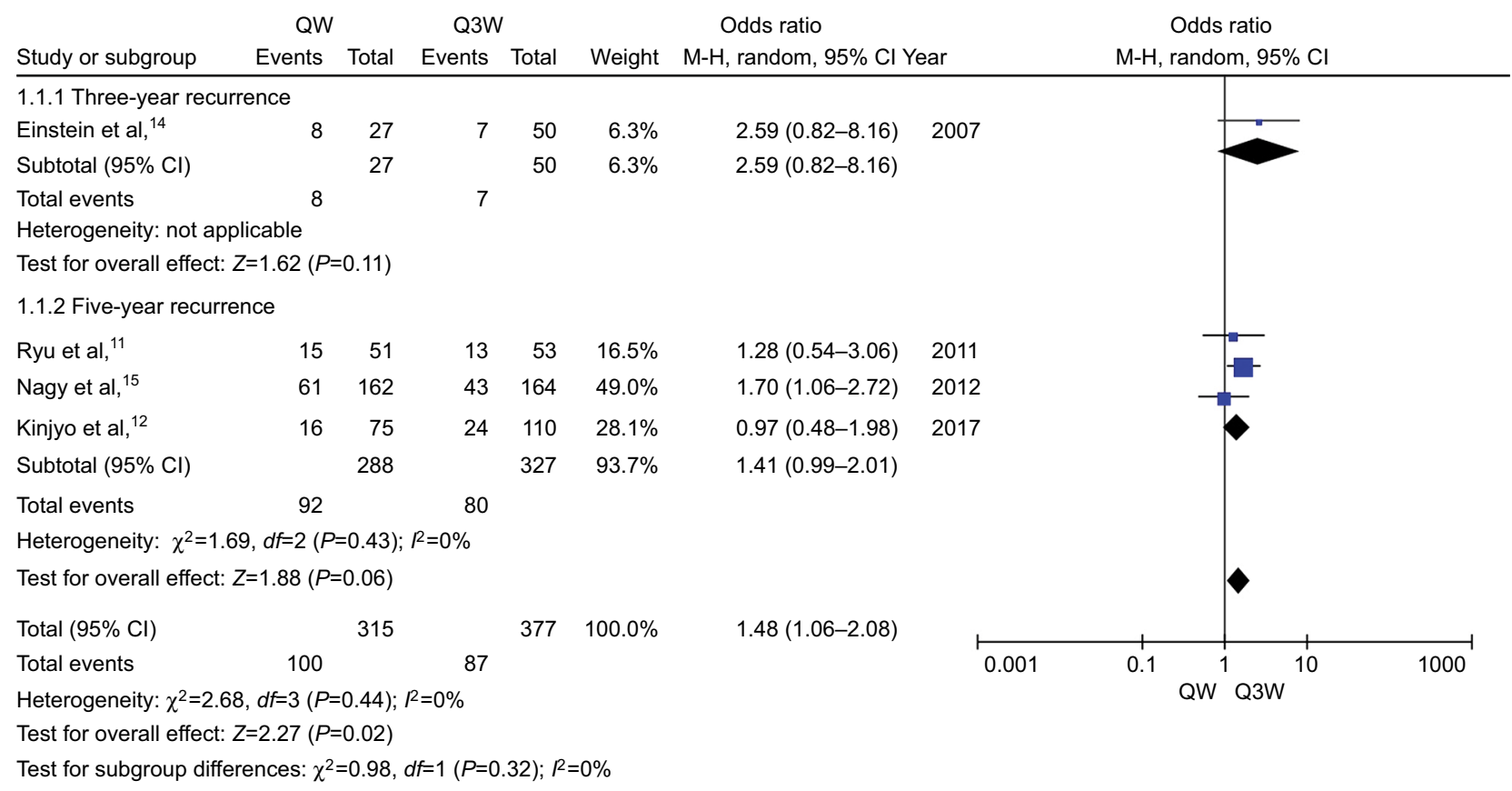

Figure 3 Meta-analysis evaluating the recurrence of weekly single cisplatin or triweekly cisplatin alone combined with radiotherapy. Abbreviations: QW, weekly; Q3W, triweekly. 


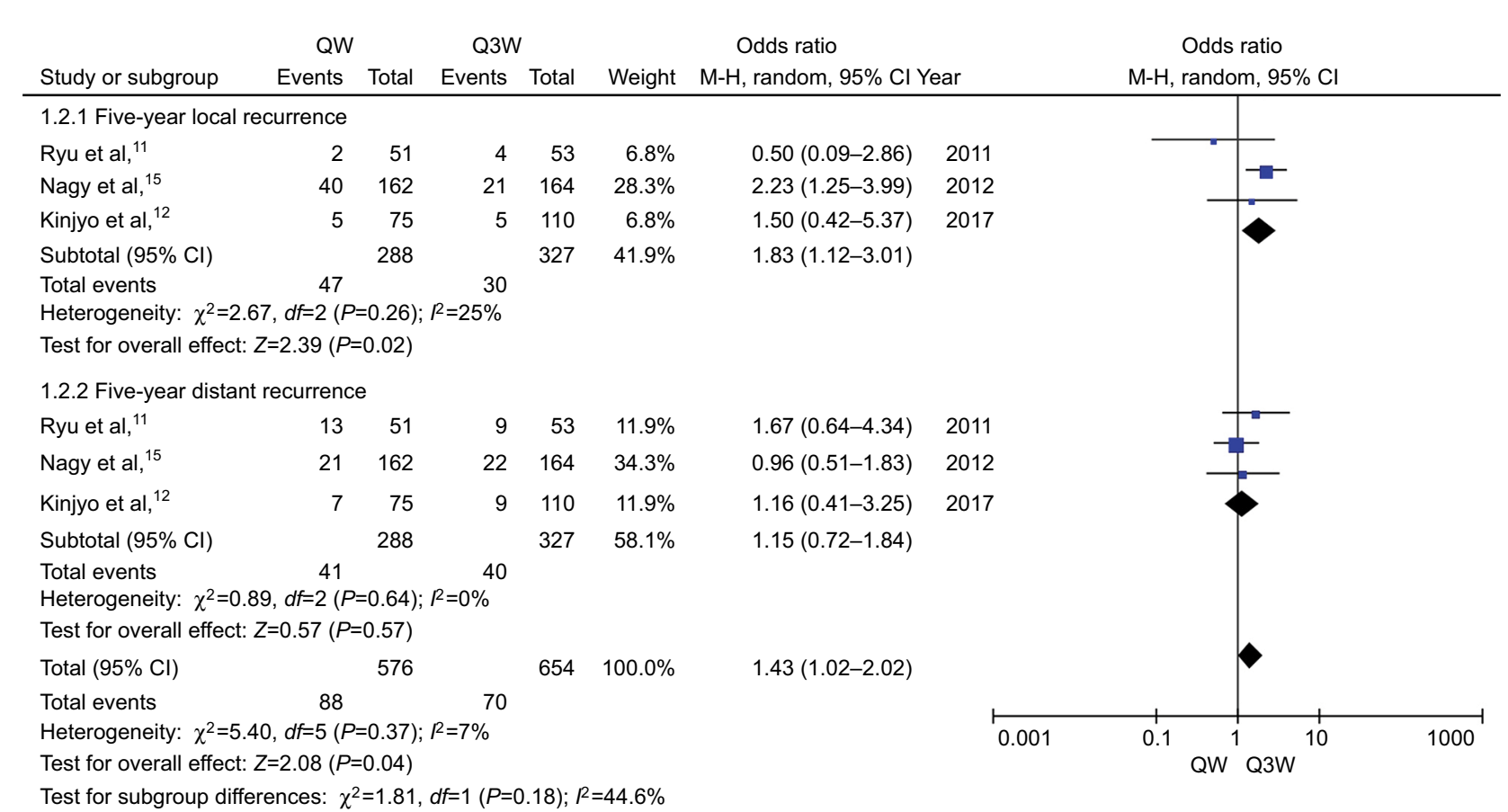

Figure 4 Meta-analysis evaluating the local relapse and distant recurrence of weekly single cisplatin or triweekly cisplatin alone combined with radiotherapy. Abbreviations: QW, weekly; Q3W, triweekly.

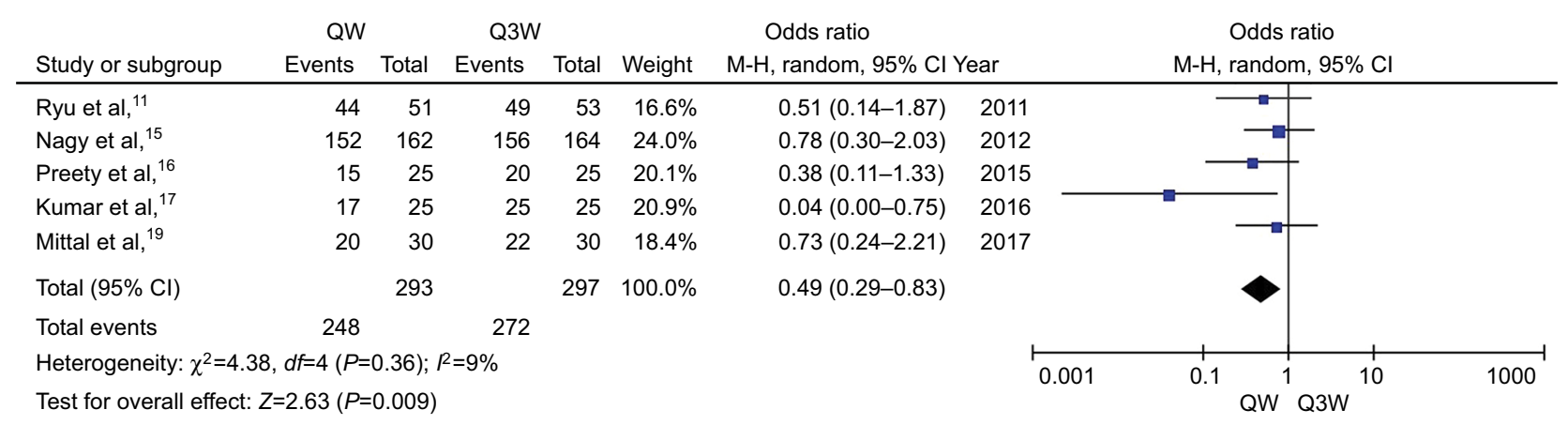

Figure 5 Meta-analysis evaluating the compliance of weekly single cisplatin or triweekly cisplatin alone combined with radiotherapy. Abbreviations: QW, weekly; Q3W, triweekly.

meta-analysis of compliance ( $\mathrm{n}=5$ studies) determined that triweekly CDDP plus RT was associated with a $14 \%$ increased risk of compliance compared with weekly CDDPbased CT plus RT (OR, 0.49; 95\% CI: $0.29-0.83 ; P=0.009$; Figure 5). Early or late toxicities of RT and chemotherapy might be the main influences.

We only chose 2 and 3 trials for the meta-analysis of leucopenia and vomiting, respectively, for assessment of acute adverse events because of differences in evaluation methods and underreporting of data in the included publications. Triweekly CDDP plus RT was associated with a $100 \%$ increased risk of leucopenia compared with weekly CDDP-based CT plus RT (OR, $0.30 ; 95 \%$ CI: 0.10-0.92; $P=0.03$; Figure 6). No significant difference was observed between the 2 regimens of CDDP-based CCRT with respect to incidence of vomiting (OR, 1.14; 95\% CI: 0.60-2.15; $P=0.69$; Figure 6).

\section{Risk of bias}

The hardboard tests for all the indices did not show any evidence of publication bias (all $P>0.05$ ) (details can be seen in Supplementary materials of Harbord tests [Tables S1-S6]).

\section{Discussion}

Weekly CDDP treatment regimen was recommended by National Comprehensive Cancer Network guidelines, based on the results of 5 randomized trials 2-6 conducted during 


\begin{tabular}{|c|c|c|c|c|c|c|c|c|c|}
\hline \multirow[b]{2}{*}{ Study or subgroup } & \multicolumn{2}{|l|}{ QW } & \multicolumn{2}{|c|}{ Q3W } & \multicolumn{2}{|r|}{ Odds ratio } & \multirow{2}{*}{\multicolumn{2}{|c|}{$\begin{array}{l}\text { Odds ratio } \\
\mathrm{M}-\mathrm{H} \text {, random, } 95 \% \mathrm{Cl}\end{array}$}} & \\
\hline & Events & Total & Events & Total & Weight & $\mathrm{M}-\mathrm{H}$, random, 95\% Cl & & & \\
\hline \multicolumn{10}{|l|}{ 1.1.1 Leucopenia } \\
\hline Kumar et al, ${ }^{17}$ & 8 & 25 & 11 & 53 & $22.1 \%$ & $0.60(0.19-1.90)$ & 2016 & - & \\
\hline Kinjyo et al, ${ }^{12}$ & 26 & 75 & 81 & 110 & $27.9 \%$ & $0.19(0.10-0.36)$ & 2017 & & \\
\hline Subtotal $(95 \% \mathrm{Cl})$ & & 100 & & 135 & $50.0 \%$ & $0.30(0.10-0.92)$ & & & \\
\hline Total events & 34 & & 92 & & & & & & \\
\hline \multicolumn{10}{|c|}{$\begin{array}{l}\text { Heterogeneity: } \tau^{2}=0.43 ; \chi^{2}=2.92, d f=1(P=0.09) ; R^{2}=66 \% \\
\text { Test for overall effect: } Z=2.11(P=0.03)\end{array}$} \\
\hline \multicolumn{10}{|c|}{ 1.1.2 Vomiting } \\
\hline Ryu et al, ${ }^{11}$ & 12 & 51 & 11 & 53 & $24.7 \%$ & $1.17(0.46-2.97)$ & 2011 & ـ & \\
\hline Kumar et al, ${ }^{17}$ & 25 & 25 & 25 & 25 & & Not estimable & 2016 & & \\
\hline Panda et al ${ }^{18}$ & 24 & 41 & 23 & 41 & $25.3 \%$ & $1.10(0.46-265)$ & 2017 & - & \\
\hline Subtotal $(95 \% \mathrm{Cl})$ & & 117 & & 119 & $50.0 \%$ & $1.14(0.60-2.15)$ & & & \\
\hline Total events & 61 & & 59 & & & & & & \\
\hline \multicolumn{10}{|c|}{ Heterogeneity: $\tau^{2}=0.00 \chi^{2}=0.01, d f=1(P=0.92) ; P^{2}=0 \%$} \\
\hline \multicolumn{10}{|c|}{ Test for overall effect: $Z=0.40(P=0.69)$} \\
\hline Total $(95 \% \mathrm{Cl})$ & & 217 & & 254 & $100.0 \%$ & $0.60(0.22-1.61)$ & & & \\
\hline Total events & 95 & & 151 & & & & & & \\
\hline \multicolumn{10}{|c|}{ Heterogeneity: $\tau^{2}=0.81 ; \chi^{2}=15.34, d f=3(P=0.002) ; P^{2}=80 \%$} \\
\hline \multicolumn{7}{|c|}{ Test for overall effect: $Z=1.01(P=0.31$} & 0.001 & 0.1 & 1000 \\
\hline \multicolumn{7}{|c|}{ Test for subgroup differences: $\chi^{2}=4.11, d f=1(P=0.04) ; p^{2}=75.7 \%$} & & QW Q3W & \\
\hline
\end{tabular}

Figure 6 Meta-analysis evaluating the acute adverse of weekly single cisplatin or triweekly cisplatin alone combined with radiotherapy. Abbreviations: QW, weekly; Q3W, triweekly.

the 1990s. However, in an RCT by Ryu et al triweekly single CDDP chemotherapy concurrent with RT was associated with better 5-year survival and lower incidence of hematological toxicity compared with the conventional weekly CDDP in patients with LACC. A meta-analysis in 2014 showed better outcomes in patients with LACC treated with CDDP-based plus another drug combined with RT. However, we found that 4 out of the 8 articles included in this meta-analysis used triweekly CDDP in combination with another drug in the experiment group. Only 2 of the 8 trials used weekly CDDP regimen as part of doublet chemotherapy. Therefore, whether the triweekly CDDP regimen in the CCRT itself contributed to the good outcomes is questionable. Two meta-analyses compared concurrent weekly CDDP vs triweekly CDDP in combination with RT for treatment of CC. Both these meta-analyses suggested the superiority of weekly CDDP regimen based only on the lower incidence of hematological toxicity. ${ }^{9,10}$ Nevertheless, other drugs were also used in most of the triweekly regimens in addition to CDDP, which may have influenced the outcomes. Therefore, we compared the efficacy and side effects between weekly and triweekly CDDP-alone regimen.

In this meta-analysis, we found that triweekly CDDP (20 $\mathrm{mg} / \mathrm{m}^{2}$ for 5 days or $75 \mathrm{mg} / \mathrm{m}^{2}$ ) alone combined with RT was associated with a lower rate of local recurrence and better compliance compared with weekly CDDP $\left(40 \mathrm{mg} / \mathrm{m}^{2}\right)$ plus RT in patients with LACC. The incidence of hematological toxicity was higher in the triweekly CDDP arm, which is similar to the findings of a previous meta-analysis. Besides, the 5-year OS was relatively better in the triweekly CDDP arm $(P=0.06)$. In our meta-analysis, we found a higher treatment completion rate among patients treated with triweekly CDDP regimen. Our findings with respect to treatment compliance are consistent with that of Einstein et al; ${ }^{14}$ however, triweekly CDDP regimen was largely used for hospitalized patients with poor general physical condition, while outpatients with good physical condition always received weekly CDDP regimen. Moreover, as inpatients tend to receive prophylactic medications, we excluded the data from this study from the meta-analysis of compliance and adverse events. We still observed a better treatment completion rate and the higher incidence of hematological toxicity in the triweekly CDDP arm. Two factors may explain the lower local recurrence and relatively better 5-year survival. First, the higher the peak concentration of CDDP, the better are the outcomes to a certain degree. Although the dose-response slope of CDDP is not steep, the response of tumor to CDDP has been shown to increase with increase in the peak concentration of CDDP up to $100 \mathrm{mg} / \mathrm{m}^{2}{ }^{20,21}$ The improved or sustained high peak blood levels of CDDP may be more effective not only in enhancing the synergy of chemoradiation but also in eliminating micrometastases, with the resultant decrease in local failure, and eventual survival benefit. Another factor may be the synergistic effect of high peak concentrations 
of CDDP with brachytherapy during the last few cycles of chemotherapy. Whether CDDP acts as a radiosensitizer during brachytherapy needs further study.

One criticism of our analysis could emanate from our inclusion of 1 trial that included some patients who received surgery after CCRT. In the study by Nagy, 5-year local relapse-free survival in the triweekly arm (87\%) was significantly superior than that in the weekly CDDP arm (77\%) $(P<0.01)$. No statistically significant differences were observed with respect to OS (QW vs Q3W; 72\% vs 78\%; $P=0.14$ ) and disease-free survival (DFS) (QW vs Q3W; $69 \%$ vs $73 \% ; P=0.09$ ). However, patients who underwent surgery had better OS, DFS, and local relapse-free survival. Surgery may improve outcomes of CCRT for LACC; however, it is worth noting that only patients who showed good response received surgery. Few studies have analyzed the role of surgery in advanced stages at present. Another point of contention is that we did not make a further subgroup to analyze the difference between the completion rates for chemotherapy and RT. Pelvic radiation therapy in patients with $\mathrm{CC}$ may cause acute radiation enteritis, radio-cystitis, and radiodermatitis. Severe diarrhea and urinary tract symptoms may also delay the treatment process.

Our study does have some limitations. First, both prospective and retrospective studies were included in the metaanalysis. More favorable characteristics and end points were chosen for analysis. In addition, the diversity of methods used to assess treatment outcomes, such as acute adverse events led to less data inclusion. Therefore, we only selected leucopenia and vomiting as the parameters for assessment of side effects of CCRT. Additionally, some of the included studies were conducted in developing countries with limited medical facilities and trained personnel. Differences with respect to dose and duration of RT may also have influenced our results. However, almost $90 \%$ of deaths from CC occur in the developing world; India alone accounts for about $25 \%$ of the total cases. ${ }^{1}$ Finally, only published literature was included in this meta-analysis, and lack of individual patient data prevented us from adjusting for the confounding influence of disease- and patient-related variables on the treatment effect.

To our knowledge, this is the first systematic review that compares the efficiency and adverse events associated with single agent triweekly CDDP plus RT and weekly CDDP alone plus concurrent RT in patients with LACC. We found that the lower rate of local relapse and the tendency for better OS probably occurs at the cost of more side effects. We recommend triweekly single CDDP regimen over weekly CDDP-alone regimen for CCRT in patients with LACC.

\section{Acknowledgment}

This study was sponsored partly by Research and Development Key Program of Jiangsu Province (Grant BE2015645) and Grant Z201413 from Scientific Research Project of Health Department of Jiangsu Province and Suzhou Key Medical Center Program (Grant szzx201506).

\section{Disclosure}

The authors report no conflicts of interest in this work.

\section{References}

1. Fitzmaurice C, Dicker D, Pain A, et al. The global burden of cancer 2013. JAMA Oncol. 2015;1(4):505.

2. Lin J. Cisplatin, radiation, and adjuvant hysterectomy compared with radiation and adjuvant hysterectomy for bulky stage IB cervical carcinoma. N Engl J Med. 1999;340(15):1154-1161.

3. Morris M, Eifel PJ, Lu J, et al. Pelvic radiation with concurrent chemotherapy compared with pelvic and para-aortic radiation for high-risk cervical cancer. N Engl J Med. 1999;340(15):1137-1143.

4. Peters W, Stock R, Monk B, et al. Concurrent chemotherapy and pelvic radiation therapy compared with pelvic radiation therapy alone as adjuvant therapy after radical surgery in high-risk early-stage cancer of the cervix. J Clin Oncol. 2000;18(8):1606-1613.

5. Malfetano J. Concurrent cisplatin-based radiotherapy and chemotherapy for locally advanced cervical cancer. NEngl J Med. 1999;340(15):1144.

6. Whitney CW, Sause W, Bundy BN, et al. Randomized comparison of fluorouracil plus cisplatin versus hydroxyurea as an adjunct to radiation therapy in stage IIB-IVA carcinoma of the cervix with negative paraaortic lymph nodes: a Gynecologic Oncology Group and Southwest Oncology Group study. J Clin Oncol. 1999;17(5):1339-1348.

7. Lanciano R, Calkins A, Bundy BN, et al. Randomized comparison of weekly cisplatin or protracted venous infusion of fluorouracil in combination with pelvic radiation in advanced cervix cancer: a Gynecologic Oncology Group study. J Clin Oncol. 2005;23(33):8289-8295.

8. Petrelli F, De SA, Raspagliesi F, Lorusso D, Barni S. Radiotherapy with concurrent cisplatin-based doublet or weekly cisplatin for cervical cancer: a systematic review and meta-analysis. Gynecol Oncol. 2014;134(1):166-171.

9. Hu Y, Cai ZQ, Su XY. Concurrent weekly cisplatin versus triweekly cisplatin with radiotherapy in the treatment of cervical cancer: a metaanalysis result. Asian Pac J Cancer Prev. 2012;13(9):4301-4304.

10. Chen X, Zou H, Li H, et al. Weekly versus triweekly cisplatin-based chemotherapy concurrent with radiotherapy in the treatment of cervical cancer: a meta-analysis. Int J Gynecol Cancer. 2016;27(2):344-349.

11. Ryu SY, Lee WM, Kim K, et al. Randomized clinical trial of weekly vs. triweekly cisplatin-based chemotherapy concurrent with radiotherapy in the treatment of locally advanced cervical cancer. Int J Radiat Oncol Biol Phys. 2011;81(4):577-581.

12. Kinjyo $Y$, Nagai $Y$, Toita $T$, et al. Concurrent weekly cisplatin versus triweekly cisplatin with radiotherapy for locally advanced squamous cell carcinoma of the cervix: a retrospective analysis from a single institution. Br J Radiol. 2017;90(1076):20170241.

13. Wells GA, Shea BJ, O'Connell D, et al. The Newcastle-Ottawa Scale (NOS) for assessing the quality of non-randomized studies in metaanalysis. Appl Eng Agric. 2014;18(6):727-734.

14. Einstein MH, Novetsky AP, Garg M, et al. Survival and toxicity differences between 5-day and weekly cisplatin in patients with locally advanced cervical cancer. Cancer. 2010;109(1):48-53.

15. Nagy VM, Ordeanu C, Coza O, Alin CR, Traila A, Todor N. Randomized phase 3 trial comparing 2 cisplatin dose schedules in 326 patients with locally advanced squamous cell cervical carcinoma: long-term follow-up. Int J Gynecol Cancer. 2012;22(9):1538-1544. 
16. Preety J, Fareed K, Amit A, et al. Comparative study of weekly versus three weekly cisplatin in advanced cases of carcinoma cervix alone with radiotherapy. Journal of Evolution of Medical and Dental Sciences. 2015;4(88):15313-15320.

17. Kumar P, Sharma K, Singh DP, et al. Weekly verses tri-weekly concurrent cisplatin with radiotherapy in the treatment of cervical cancer: a study comparing efficacy and toxicity. SRMS Journal of Medical Sciences. 2017;1(2):66-72.

18. Panda N, Bag S, Samantaray S, et al. Randomised clinical trial of weekly vs. triweekly cisplatin based chemotherapy concurrent with radiotherapy in the treatment of locally advanced cervical cancer. Int $J$ Med Clin Res. 2017;5(04):20060-20064.
19. Mittal S, Chauhan A, Kaur P, et al. Comparing weekly versus three weekly schedules of cisplatinum concomitant with radical radiotherapy in locally advanced carcinoma uterine cervix. Gynecology Obstetrics. 2017;7:1.

20. Bonomi P, Blessing JA, Stehman FB, et al. Randomized trial of three cisplatin dose schedules in squamous-cell carcinoma of the cervix: a Gynecologic Oncology Group Study. J Clin Oncol. 1985;3(8):1079.

21. Thigpen T, Shingleton H, Homesley H, et al. cis-Dichlorodiammineplatinum (II) in the treatment of gynecologic malignancies: phase II trials by the Gynecologic Oncology Group. Cancer Treat Rep. 1979;63(9-10) $1549-1555$. 


\section{Supplementary materials}

1. Compliance

Metabias qwevent qwtotal q3wevent q3wtotal, Harbord graph. Note: data input format tcases tnoncases ccases cnoncases assumed. Odds ratios assumed as effect estimate of interest (Table S1).

\section{Five-year OS}

Metabias qwevent qwtotal q3wevent q3wtotal, Harbord graph.

Note: data input format tcases tnoncases ccases cnoncases assumed. Odds ratios assumed as effect estimate of interest (Table S2).

\section{Five-year recurrence}

Metabias qwevent qutotal q3wevent q3wtotal, Harbord graph. Note: data input format tcases tnoncases ccases cnoncases assumed. Odds ratios assumed as effect estimate of interest (Table S3).

\section{Subgroup-local}

Metabias qwevent qwtotal q3wevent q3wtotal, Harbord graph.

Note: data input format tcases tnoncases ccases cnoncases assumed. Odds ratios assumed as effect estimate of interest (Table S4).

\section{Subgroup-distance}

Metabias qwevent qwtotal q3wevent q3wtotal, Harbord graph.

Note: data input format tcases tnoncases ccases cnoncases assumed. Odds ratios assumed as effect estimate of interest (Table S5).

\section{Vomiting}

Metabias qwevent qwtotal q3wevent q3wtotal, Harbord graph.

Note: data input format tcases tnoncases ccases cnoncases assumed. Odds ratios assumed as effect estimate of interest (Table S6).

Table SI Harbord's modified test for small-study effects

\begin{tabular}{lllllll}
\hline Z/sqrt(V)| & Coefficient & Standard error & $\boldsymbol{t}$ & $\mathbf{P}>|\mathbf{t}|$ & $\mathbf{9 5 \%} \mathbf{C l}$ \\
\hline sqrt(V)| & 0.1361856 & 0.0857646 & 1.59 & $0.21 \mathrm{I}$ & -0.1367557 & 0.409127 \\
bias | & -0.8858072 & 0.3188885 & -2.78 & 0.069 & -1.900653 & 0.1290382 \\
\hline
\end{tabular}

Notes: Regress $Z /$ sqrt( $(V)$ on sqrt( $(V)$ where $Z$ is efficient score and $V$ is score variance. Number of studies $=5$. Root MSE $=0.2907$. Test of H0: no small-study effects. $P=0.069$.

Table S2 Harbord's modified test for small-study effects

\begin{tabular}{lllllll}
\hline Z/sqrt(V)| & Coefficient & Standard error & $\boldsymbol{t}$ & $\mathbf{P}>|\mathbf{t}|$ & $\mathbf{9 5 \%} \mathbf{C l}$ \\
\hline sqrt(V)| & 0.1511562 & 0.363759 & 0.42 & 0.749 & -4.47084 & 4.773152 \\
bias | & -1.166011 & 1.7125 & -0.68 & 0.619 & -22.92538 & 20.59336 \\
\hline
\end{tabular}

Notes: Regress $Z /$ sqrt( $(V)$ on sqrt( $(V)$ where $Z$ is efficient score and $V$ is score variance. Number of studies $=3$. Root MSE $=0.6543$. Test of H0: no small-study effects. $P=0.619$.

Table S3 Harbord's modified test for small-study effects

\begin{tabular}{lllllll}
\hline Z/sqrt(V) | & Coefficient & Standard error & $\boldsymbol{t}$ & $\mathbf{P}>|\mathbf{t}|$ & $\mathbf{9 5 \%} \mathbf{C l}$ \\
\hline sqrt(V)| & 0.6797789 & 0.3750117 & 1.81 & 0.321 & -4.085196 & 5.444754 \\
bias | & -1.529269 & 1.248377 & -1.23 & 0.436 & -17.3914 & 14.33286
\end{tabular}

Notes: Regress $Z / s q r t(V)$ on sqrt( $V)$ where $Z$ is efficient score and $V$ is score variance. Number of studies $=3$. Root MSE $=0.5805$. Test of H0: no small-study effects. $P=0.436$.

Table S4 Harbord's modified test for small-study effects

\begin{tabular}{lllllll}
\hline Z/sqrt(V) | & Coefficient & Standard error & $\boldsymbol{t}$ & $\boldsymbol{P}>|\mathbf{t}|$ & $\mathbf{9 5 \%} \mathbf{C l}$ \\
\hline sqrt(V) | & 1.10397 & 0.3805126 & 2.90 & $0.21 \mathrm{I}$ & -3.730901 & 5.938841 \\
bias | & -1.611068 & 0.8948439 & -1.80 & 0.323 & -12.98114 & 9.759002 \\
\hline
\end{tabular}

Notes: Regress $Z / s q r t(V)$ on sqrt( $V)$ where $Z$ is efficient score and $V$ is score variance. Number of studies $=3$. Root $M S E=0.6977$. Test of H0: no small-study effects. $P=0.323$. 
Table S5 Harbord's modified test for small-study effects

\begin{tabular}{lllllll}
\hline Z/sqrt(V)| & Coefficient & Standard error & $\boldsymbol{t}$ & $\mathbf{P}>|\mathbf{t}|$ & $\mathbf{9 5 \%} \mathbf{C l}$ \\
\hline sqrt(V)| & $-0.49560 \mathrm{I}$ & $0.587392 \mathrm{I}$ & -0.84 & 0.554 & -7.959125 & 6.967923 \\
bias | & 1.508144 & 1.425639 & 1.06 & 0.482 & 16.60632 & $19.6226 \mathrm{I}$ \\
\hline
\end{tabular}

Notes: Regress $Z /$ sqrt( $(V)$ on sqrt( $(V)$ where $Z$ is efficient score and $V$ is score variance. Number of studies $=3$. Root MSE $=0.5239$. Test of H0: no small-study effects. $P=0.482$.

Table S6 Harbord's modified test for small-study effects

\begin{tabular}{lllllll}
\hline Z/sqrt(V) | & Coef. & Standard error & $\boldsymbol{T}$ & $\mathbf{P}>|\mathbf{t}|$ & $\mathbf{9 5 \%} \mathbf{C l}$ \\
\hline sqrt(V) | & -0.3129438 & 0.3623589 & -0.86 & 0.547 & -4.91715 & 4.291262 \\
bias | & 0.9045132 & 0.9019209 & 1.00 & 0.499 & -10.55548 & 12.3645 \\
\hline
\end{tabular}

Notes: Regress $Z / s q r t(V)$ on $\operatorname{sqrt}(V)$ where $Z$ is efficient score and $V$ is score variance. Number of studies $=3$. Root MSE $=0.1456$. Test of H0: no small-study effects. $P=0.499$.

Cancer Management and Research is an international, peer-reviewed open access journal focusing on cancer research and the optimal use of preventative and integrated treatment interventions to achieve improved outcomes, enhanced survival and quality of life for the cancer patient. The manuscript management system is completely online and includes a very quick and fair peer-review system, which is all easy to use. Visit http://www.dovepress.com/testimonials.php to read real quotes from published authors. 